\title{
SGLT-2 Inhibitors: Drug Selection by Means of the System of Objectified Judgement Analysis Method
}

\author{
Robert Janknegt $^{1 *}$, Mark Reinders ${ }^{1}$ and Rutger Bianchi ${ }^{2}$ \\ ${ }^{1}$ Hospital Pharmacist, Zuyderland Medical Centre, The Netherlands \\ ${ }^{2}$ Internist, Endocrinologist, Zuyderland Medical Centre, The Netherlands \\ *Corresponding Author: Robert Janknegt, Hospital Pharmacist, Zuyderland \\ Medical Centre, The Netherlands.
}

Received: February 26, 2021

Published: June 11, 2021

(C) All rights are reserved by Robert

Janknegt., et al.

\section{Abstract}

Objectives: The increasing number of antidiabetic drugs makes it almost impossible to have sufficient knowledge of each individual medicine and device, especially for general practitioners.

Reducing the number of different antidiabetics based on rational criteria, allows physicians and pharmacists to build experience with a more limited set of medicines and to optimise patient information.

Methods: In this study SGLTs are compared by means of the SOJA method.

The following selection criteria were applied: ease of use, available dosage forms, clinical efficacy, documented effects on clinical endpoints, safety, tolerability, drug interactions and documentation.

Results: Some differences in scores were found between canagliflozin, dapagliflozin, empagliflozin and ertugliflozin. Ertugliflozin showed the lowest score, mostly because of its more limited clinical evidence and documentation. Empagliflozin and dapagliflozin showed the highest scores. These medicines are well documented concerning clinical endpoints, including heart failure and safety.

Acquisition cost was not taken into account, because this varies with time. In practice acquisition cost is of course an important selection criterion, especially because there are no major differences between the medicines from a clinical perspective. Exclusion of this criterion also makes this comparison more internationally applicable.

Conclusions: Empagliflozin and dapagliflozin showed the highest scores, mainly because of their documented effects on clinically relevant endpoints and safety.

Keywords: Dapagliflozin; Empagliflozin; SOJA Method

\section{Introduction}

Diabetes

The prevalence of type 2 diabetes in Europe is at least $2-3 \%$ and increases significantly in patients over 70 years of age.

Microvascular [retinopathy, nephropathy and neuropathy] and macrovascular complications such as ischemic heart disease, cerebrovascular accident and peripheral arterial vascular disease frequently occur. Microvascular complications are probably due primarily to hyperglycemia, while macrovascular complications are more related to the interaction between hyperglycemia, insulin resistance, dyslipidemia and hypertension $[1,2]$. 
The UK Prospective Diabetes Study [UKPDS] has been studying various aspects of the natural course and treatment of type 2 diabetes mellitus since the 1970s.

In one of the investigations [UKPDS 29], the incidence of CVA was investigated. The average study duration was 7.9 years. CVA was seen in $2.6 \%$ of patients. The main risk factors were age, male sex and hypertension [3]. The major risk factors for developing coronary heart disease were elevated LDL cholesterol, decreased HDL cholesterol, elevated triglycerides, elevated systolic blood pressure, elevated HbA1c, increased fasting blood glucose and smoking [4].

For the treatment of type 2 diabetes mellitus, a number of oral agents are available with different mechanisms of action: sulfonylurea derivatives and meglitinides [increase of insulin secretion through the pancreas], metformin [primarily inhibition of hepatic glucose production and partially inhibited glucose uptake in peripheral tissues] ,aAlpha-glucosidase inhibitors [delayed absorption of intestinal monosaccharides] and thiazolidinediones [reduction of insulin resistance and enhancement of insulin effects on glucose metabolism] [1,2,5-12]. DPP-4 inhibitors, GLP inhibitors and SGLT-2 inhibitors were introduced more recently.

The differences between the various modes of action, side effects, demonstrated effect on clinically relevant endpoints, drug interactions, price and documentation are significant, which necessitates weighted weighing of pros and cons of the various medicines.

Sodium-glucose cotransporter-2 [SGLT2] inhibitors are the newest class of oral antihyperglycemic agents available for the treatment of diabetes mellitus type 2 . SGLT2 inhibitors act by reducing renal tubular glucose reabsorption, producing a reduction in blood glucose without stimulating insulin release. Other benefits may include favorable effects on blood pressure and weight.

This article focuses on drug selection within this class of medicines, using the SOJA method.

\section{Methodology}

The System of Objectified Judgement Analysis [SOJA] method is a model for rational drug selection. The relevant selection criteria for a certain group of drugs are defined and judged by a panel of experts and each selection criterion is given a relative weight. The more important that a selection criterion is considered, the higher the relative weight that is given to that criterion. The ideal properties for each selection criterion are determined and each drug is scored as a percentage of the score of the ideal drug for all selection criteria. The drugs with the highest total score are most suitable for formulary inclusion [13].

After the authors had weighted the criteria, Medline and the Cochrane database were searched and references from review articles obtained. A request was sent to all companies to submit all articles on their medicine that they considered relevant for the matrix. It was explicitly stated that the project was not supported by any pharmaceutical companies.

The evaluation of criteria in the SOJA method is highly standardised in order to promote unbiased judgement of drugs from various pharmacotherapeutic categories based on clinically relevant criteria. There will of course always be room for debate whether or not the correct scoring system was used for each criterion and judgement may be arbitrary for most, if not all, criteria. This is the case with any method used to quantify properties of drugs. The SOJA method is intended as a tool for rational drug decision making, forcing clinicians and pharmacists to include all relevant aspects of a certain group of drugs, thereby preventing formulary decisions being based on only one or two criteria. Besides this, possible "hidden criteria" are excluded from the decision making process. The outcome of this study should be seen as the basis for discussions within formulary committees and not as an absolute truth.

This analysis is limited to the subpopulation of overweight patients. This subpopulation comprises $85 \%$ of the total type 2 diabetes population. In these patients, insulin resistance plays an important role, as opposed to patients without obesity.

This analysis focuses on the SGLT-2 inhibitors:

- Canagliflozin [Invokana]

- Dapagliflozin [Forxiga]

- Empagliflozin [Jardiance]

- Ertugliflozin [Steglatro]

\section{Selection criteria}

The following selection criteria are applied 


\begin{tabular}{|l|c|}
\hline \multicolumn{1}{|c|}{ Criterion } & Relative weight \\
\hline Ease of use & 50 \\
\hline Available dosage forms & 50 \\
\hline Efficacy & 200 \\
\hline $\begin{array}{l}\text { Documented effects on clinically relevant } \\
\text { endpoints }\end{array}$ & 250 \\
\hline Safety & 200 \\
\hline Tolerability & 100 \\
\hline Interactions & 50 \\
\hline Documentation & 100 \\
\hline Total & 1000 \\
\hline
\end{tabular}

Table a

\begin{tabular}{|l|c|}
\hline & Score \\
\hline $\begin{array}{l}\text { No need to stop existing treatment in case of } \\
\text { decreased renal function }\end{array}$ & $100 \%$ \\
\hline Stopping at eGFR $<30 \mathrm{ml} / \mathrm{min}$ & $75 \%$ \\
\hline Stopping at eGFR $<45 \mathrm{ml} / \mathrm{min}$ & $50 \%$ \\
\hline Stopping at eGFR $<60 \mathrm{ml} / \mathrm{min}$ & $25 \%$ \\
\hline Contra-indicated & $0 \%$ \\
\hline
\end{tabular}

Table c

\section{Applicability}

Availability of different forms of administration

This was scored as follows:

Ease of use

This criterion contains 2 subcriteria:

- $\quad$ Dosage frequency: $80 \%$

- Applicability in renal function impairment : : $20 \%$.

Dosage frequency

Dosage frequency plays an important role in patient compliance. Compliance is not usually a problem in patients taking the drugs once or twice daily, but decreases considerably in the event that 3-4 dosages are to be taken daily. The method of evaluation of this criterion corresponded with that of all of the other SOJA scores.

\begin{tabular}{|l|c|}
\hline \multicolumn{1}{|c|}{ Frequency } & Score \\
\hline Once daily & $100 \%$ \\
\hline One to two times daily & $90 \%$ \\
\hline Twice daily & $80 \%$ \\
\hline Two to three times daily & $60 \%$ \\
\hline Three times daily & $40 \%$ \\
\hline Four times daily & $10 \%$ \\
\hline
\end{tabular}

Table b

\begin{tabular}{|l|c|}
\hline & Score \\
\hline One strength only & $20 \%$ \\
\hline Two or more strengths & $40 \%$ \\
\hline $\begin{array}{l}\text { Combination tablet with one other class of } \\
\text { antidiabetics }\end{array}$ & $+20 \%$ \\
\hline $\begin{array}{l}\text { Combination tablet with two other classes of } \\
\text { antidiabetics }\end{array}$ & $+40 \%$ \\
\hline $\begin{array}{l}\text { Combination tablet with three or more other classes } \\
\text { of antidiabetics }\end{array}$ & $+60 \%$ \\
\hline
\end{tabular}

Table d

\section{Clinical efficacy}

Clinical efficacy is by definition a very important selection criterion for each group of drugs. The relative efficacy of the various [classes of] medicines used for maintenance treatment of diabetes mellitus was determined using [preferably double-blind] randomised comparative studies between these drugs in the first instance.

If these studies were not available, results from randomised placebo-controlled studies or [double-blind or open-label] studies with other medicines included in this analysis were also taken into consideration.

There is a large number of placebo controlled and directly comparative clinical trials conducted with SGLT- 2 inhibitors. [references available on request from the authors] Intensive treatment 
of type 2 diabetes mellitus, regardless of the chosen agent, leads to a significant improvement of "surrogate markers" such as HbA1c [14].

Glycemic control [HbA1c, fasting glucose levels]

HbA1c is an important "marker" for the risk of microvascular complications. In this article $\mathrm{HbA} 1 \mathrm{c}$ is expressed as \%. HbA1c is also expressed as $\mathrm{mmol} / \mathrm{mol}$. Seven \% equals $53 \mathrm{mmol} / \mathrm{mol}$, and every \% point counts for $11 \mathrm{mmol} / \mathrm{mol}$, so $8 \%$ equals $64 \mathrm{mmol} /$ mol and so on. The maintenance of HbA1c below 7\% was found to be associated with a reduced incidence of microvascular complications, whereas they occur more frequently at HbA1c values above $10 \%[15,16]$. In an analysis of the effects of HbA1c on the incidence of diabetes complications, UKPDS 35), every $1 \%$ reduction in $\mathrm{HbA} 1 \mathrm{c}$ showed a $21 \%$ reduction in total diabetes complications, $21 \%$ diabetes-related mortality, $14 \%$ reduction of myocardial infarction and $37 \%$ of microvascular complications [17]. In the UKPDS 47 study, a relationship was found between HbA1c $[<6.3$ or $>$ 7.6\%] and fatal or non-fatal myocardial infarction, but not with CVA [18]. In the more recent UKPDS 66 study, a relationship between HbA1c and the subsequent occurrence of fatal myocardial infarction or fatal CVA [19] was found.

Determining HbA1c is a better "parameter" for chronic blood glucose control than the fasting blood glucose measurements, which are snapshots [20].

It is important that the results of direct comparisons between two or more agents are used in the analysis of the effects of the blood glucose lowering agents. The results of placebo-controlled research cannot be used to make statements about the mutual effectiveness of the various medicines. The effects on HbA1c depend on the baseline HbA1c [higher reduction at higher baseline] and the history of the use of blood glucose lowering agents prior to the study [stronger reduction in "naive" patients] [1,2,21,22].

Effects on insulin resistance

Type 2 diabetes mellitus is a heterogeneous condition charac- terized by abnormalities in the beta cells of the pancreas and in peripheral tissues, such as skeletal muscle and fat tissue. At least three metabolic disorders play a role in the development of hyperglycemia in patients with type 2 diabetes mellitus: decreased insulin secretion in response to glucose, increased glucose production in the liver and a reduced insulin-dependent glucose uptake in peripheral tissues [23-26]. The last two abnormalities are defined as insulin resistance. Insulin resistance can be treated by enhancing insulin activity, increasing glucose consumption in peripheral tissues, reducing gluconeogenesis and glycogenolysis in the liver and reducing lipolysis in the fat cells. Insulin resistance occurs in the first phase of the disease and can contribute to the progression of the disease and depletion of the beta cells. Insight into the importance of insulin resistance and insulin resistance syndrome [dyslipidemia, increased chance of intravenous clotting, endothelial dysfunction and hypertension] has grown [1,23,27] in recent years, and insulin resistance is now believed to play a central role in developing Name macrovascular complications of type 2 diabetes mellitus. Medicines that can reduce insulin resistance work, to some extent, causal $[23,24]$.

Of these effects only effects on $\mathrm{HbA} 1 \mathrm{c}$ [70\% of relative weight] and insulin resistance [ $30 \%$ of relative weight] were scored.

\section{Documented effects of clinically relevant endpoints}

The following effects were taken into consideration:

- Effect on macrovascular complications [myocardial infarction, stroke, peripheral vascular disease or acute death].

- Effects on microvascular complications [neuropathy, retinopathy, nephropathy].

This was scored as follows:

Safety

Rare, dangerous side effects

The extent and the severity of adverse effects is another important selection criterion for drugs. A distinction was made between

\begin{tabular}{|l|c|c|c|c|c|}
\hline & Macrovascular complications & Neuropathy & Retinopathy & Nephropathy & Score \\
\hline Max score & $60 \%$ & $10 \%$ & $10 \%$ & $20 \%$ & $100 \%$ \\
\hline
\end{tabular}

Table e 
"minor" side effects, such as gastrointestinal disturbances or skin reactions, occurring in clinical trials [scored under tolerability] and severe or even life-threatening adverse reactions observed with large scale use of the drugs.

\section{Tolerability}

The extent and the severity of adverse effects is another important selection criterion for drugs. A distinction was made between "minor" side effects, such as gastrointestinal disturbances or skin reactions, occurring in clinical trials and severe or even life-threatening adverse reactions observed with large scale use of the drugs [scored under safety]. The evaluation of the "minor" adverse effects was based on results of double blind comparative clinical studies.

\section{Drug interactions}

This criterion is of importance in formulary decision making as the majority of patients treated with diabetes will take other medications as well. Drug interactions may result in an increased or reduced clinical efficacy of the antidiabetic medicine in question or in a reduction of the clinical efficacy of the other drug, with which the interaction occurs. Interactions may also give rise to increased toxicity of one or both compounds. The more frequent these interactions occur and the more serious the consequences are, the lower the score for the drug in question.

\section{Documentation}

The first two sub criteria are indicative of the overall clinical documentation of the drugs in randomised controlled clinical studies. A large number of clinical studies and a large number of patients included in these studies leave no doubt about the clinical efficacy and safety of this drug in the studied population. The latter two criteria are indicative of the overall clinical experience with the drug. These sub criteria may introduce a bias to the advantage of older drugs, but this is done intentionally. The safety of a newly introduced drug cannot be guaranteed from the results of clinical studies, in which only a relatively small number of patients were included and most patients at risk for the development of adverse reactions [e.g. patients with diminished renal function] were excluded. Both the number of patients that has been treated on a worldwide basis and the period that a certain drug has been available are of importance, as it may take time until adverse reactions occur.
The method of evaluation of this criterion was identical to that of all of the other SOJA scores. The score includes the following aspects:

- $\quad$ The number of comparative studies: $25 \%$

- $\quad$ The number of patients in these studies : $25 \%$

- The number of years on the market: $25 \%$

- The number of patient days worldwide: $25 \%$

The number of comparative studies

Five percent of the maximum score was assigned for each study of a specific drug. As a result, the score for 20 studies is $100 \%$.

\section{The number of patients in these studies}

For every 10 patients participating in these studies $1 \%$ of the maximum score was assigned. As a result, the score for 1000 patients is $100 \%$.

\section{The number of years on the market}

Every year a certain drug has been on the market represents $10 \%$ of the score. If a drug has been on the market for at least 10 years, the score is $100 \%$.

\section{Number of patient days worldwide}

Everyone million patient days of experience represents $1 \%$ of the score. If the number of patient days of experience exceeds 100 million, the score is $100 \%$.

\section{Results}

\section{Ease of use}

\section{Dosage frequency}

The score for dosage frequency is expressed below.

\begin{tabular}{|l|c|c|c|c|c|}
\hline & $\begin{array}{c}\text { Dosage } \\
\text { frequency }\end{array}$ & Score & $\begin{array}{c}\text { Renal func- } \\
\text { tion }\end{array}$ & Score & \\
\hline Canagliflozin & $1 \mathrm{x}$ daily & $80 \%$ & Not needed & $20 \%$ & $100 \%$ \\
\hline Dapagliflozin & $1 \mathrm{x}$ daily & $80 \%$ & $<45 \mathrm{ml} / \mathrm{min}$ & $10 \%$ & $90 \%$ \\
\hline Empagliflozin & $1 \mathrm{x}$ daily & $80 \%$ & $<45 \mathrm{ml} / \mathrm{min}$ & $10 \%$ & $90 \%$ \\
\hline Ertugliflozin & $1 \mathrm{x}$ daily & $80 \%$ & $<45 \mathrm{ml} / \mathrm{min}$ & $10 \%$ & $90 \%$ \\
\hline
\end{tabular}

Table $f$ 
User-friendly dosage forms

No user-friendly dosage forms, such as liquid or dispersible formulations are available for any compound.

\section{Food intake}

All medicines can be combined with food. In order to achieve optimal therapeutic efficacy, the intake is recommended just before or during the meal.

\section{Applicability}

Availability of different forms of administration

This resulted in the following score.

\begin{tabular}{|l|c|c|c|c|c|}
\hline & Strengths & $\begin{array}{c}\text { Subscore } \\
\text { (max } \\
\mathbf{4 0 \% )}\end{array}$ & $\begin{array}{c}\text { Combination } \\
\text { with other } \\
\text { antidiabetics }\end{array}$ & $\begin{array}{c}\text { Sub- } \\
\text { score }\end{array}$ & $\begin{array}{c}\text { Total } \\
\text { (max } \\
\mathbf{6 0 \% )}\end{array}$ \\
Score \\
\hline Canagliflozin & $\begin{array}{c}100 \mathrm{mg}, \\
300 \mathrm{mg}\end{array}$ & $40 \%$ & Metformin & $20 \%$ & $60 \%$ \\
\hline Dapagliflozin & $\begin{array}{c}5 \mathrm{mg}, 10 \\
\mathrm{mg}\end{array}$ & $40 \%$ & Metformin & $20 \%$ & $60 \%$ \\
\hline $\begin{array}{l}\text { Empa- } \\
\text { gliflozin }\end{array}$ & $\begin{array}{c}10 \mathrm{mg}, 25 \\
\mathrm{mg},\end{array}$ & $40 \%$ & Metformin & $20 \%$ & $60 \%$ \\
\hline Ertugliflozin & $\begin{array}{c}5 \mathrm{mg}, 10 \\
\mathrm{mg}\end{array}$ & $40 \%$ & Metformin & $40 \%$ & $80 \%$ \\
\hline
\end{tabular}

Table g

The combination of sitagliptin and ertugliflozin is available on the market, but is not reimbursed by health insurance companies in the Netherlands.

\section{Clinical efficacy}

It should be stressed that patient education is an important aspect of the treatment of diabetes mellitus type 2 : losing weight [by diet and exercise] is very important in reducing insulin resistance.

\section{Effects on HbA1c}

One meta-analysis studied the effects of SGLT-s inhibitors on HbA1c. The mean decrease was $0.69 \%$ compared with placebo, with canagliflozin showing the highest decrease [0.85\%]. The evidence was considered to be low quality, because of variability and evidence of publication bias [28]. Another meta-analysis also showed a slightly higher effect [difference 0.10 - 0.20\%] on HbA1c for canagliflozin compared to dapagliflozin and empagliflozin [29].

Another meta-analysis compared studies with ertugliflozin to other SGLT-2 inhibitors. The high dose of ertugliflozin [15 mg] was significantly [but slightly] more effective concerning effects on HbA1c than dapagliflozin $10 \mathrm{mg}$ or empagliflozin $25 \mathrm{mg}$ [30].

Empagliflozin showed comparable effect on $\mathrm{HbA1c}$, with a mean decrease of $0.62 \%$ compared to placebo in a meta-analysis of 15 randomised studies [31]. Another meta-analysis showed a HbA1c decrease of $0.57 \%$ for $10 \mathrm{mg}$ and $0.65 \%$ for $25 \mathrm{mg}$ empagliflozin. Empagliflozin $10 \mathrm{mg}$ was as effective as linagliptin, sitagliptin and glimepiride in direct comparative studies, whereas the $25 \mathrm{mg}$ was significantly more effective than these pooled comparators, with a $0.13 \%$ stronger reduction in $\mathrm{HbA1c}$ [32].

DPP4 inhibitors and SGLT-2 inhibitors showed similar effects on HbA1c when added to metformin plus sulfonylurea [33].

A meta-analysis showed that DPP4 inhibitors, SGLT-2 inhibitors, thiazolidinediones and sulfonylurea resulted in similar $\mathrm{HbA1c}$ reduction when added to metformin, ranging from 0.5 to $1.0 \%$ in various studies [34].

Insulin sensitivity

Few studies have been published regarding the effects of SGLT-2 inhibitorson insulin sensitivity. A positive effecton insulin sensitivity was seen in 2 clinical studies [35,36]. A positive effect is to be expected because of the weight loss induced by SGLT-2 inhibitors [37-51]. This was scored as follows.

\begin{tabular}{|l|c|c|c|}
\hline & HbA1c & $\begin{array}{c}\text { Insulin } \\
\text { Resistance }\end{array}$ & Score \\
\hline Max score & $70 \%$ & $30 \%$ & $100 \%$ \\
\hline Canagliflozin & $35 \%$ & $20 \%$ & $55 \%$ \\
\hline Dapagliflozin & $35 \%$ & $20 \%$ & $55 \%$ \\
\hline Empagliflozin & $35 \%$ & $20 \%$ & $55 \%$ \\
\hline Ertugliflozin & $35 \%$ & $20 \%$ & $55 \%$ \\
\hline
\end{tabular}

Table h

Other effects

Effects on lipids profile

Dyslipidemia occurs in $40-50 \%$ of patients with type 2 diabetes mellitus. The main features are elevated triglycerides, decreased 
HDL and normal to slightly elevated LDL cholesterol [52,53]. The UKPDS 66 study showed no relationship between the LDL/HDL ratio and the subsequent occurrence of fatal myocardial infarction or fatal CVA [12]. In the UKPDS 59 study, a relationship was found between HDL cholesterol and the occurrence of peripheral vascular disease. Any reduction of HDL with $0.1 \mathrm{mmol} / \mathrm{l}$ showed an increase of $22 \%$ risk [54].

The 10-year risk of coronary heart disease was $21 \%$ in patients with diabetes mellitus type 2 [55].

A beneficial effect on the lipid spectrum of blood glucose lowering agents is important, but in practice the vast majority of patients will need a statin to achieve optimal lipid control.

There is still no clarity about the relevance of these observations. There are no indications for relevant differences between the various compounds regarding effects on lipids.

\section{SGLT-2 inhibitors}

SGLT-2 inhibitors showed limited effects on cholesterol metabolism in clinical studies [40,42,50,56-60].

\section{Effects on blood pressure}

SGLT-2 inhibitors resulted in a decrease in systolic and diastolic blood pressure in a range of clinical and database studies [61-76]. There are no indications for relevant differences between the various compounds regarding effects on blood pressure.

\section{Documented effects of clinically relevant endpoints}

Both diabetes and high age are important risk factors for death to cardiovascular complications. Coronary heart disease is the major cause of death in type 2 diabetes patients [55]. The cardiovascular risk profile of diabetes patients is similar to that of non-diabetes individuals who are 15 years of age [77].

It is always checked whether it has been demonstrated that a significant reduction in morbidity or mortality relative to placebo or other drugs is achieved. If this is demonstrated, the size of the effect is also included in the score. In addition, it is included in the score whether it is an effect in an overall diabetes population or in a specific group, such as primary and secondary treatment, obesity, etc.
The relationship between blood glucose control and effect on macrovascular complications is complex. In a systematic review, a decreased incidence was found of non-fatal heart disease, RR 0.80 as well as the overall incidence of cardiovascular complications, RR 0.90 for intensive control over standard treatment, but not for the other endpoints, such as CVA or Cardiovascular mortality [78].

\section{SGLT-2 inhibitors}

The primary purpose of the cardiovascular safety studies is to demonstrate that the SGLT2 inhibitors are non-inferior to placebo in terms of cardiovascular morbidity and mortality. Non-inferiority means that the SGLT2 inhibitor does not cause more cardiovascular morbidity and mortality than placebo. In case the SGLT2 inhibitor was non-inferior to placebo, researchers checked whether the substance is superior. This should be included prospectively in the study protocols. Superior in this case means that the SGLT2 inhibitor causes less cardiovascular morbidity and mortality than placebo. All studies were conducted in patients at high risk for cardiovascular morbidity and mortality.

10,142 patients participated in the CANVAS and CANVAS-R study with canagliflozin. Patients had a history of cardiovascular disease or at least 2 risk factors for cardiovascular disease. The median duration of follow-up was 2.4 years [79].

The DECLARE-TIMI 53 study on dapagliflozin involved 17,160 patients. The patients had an atherosclerotic disorder in the history, or multiple risk factors for atherosclerotic disorders. The median follow-up was 4.2 years [80].

7,020 patients participated in the EMPA-REG OUTCOME study on empagliflozin. The patients had a history of cardiovascular disease. The median duration of follow-up was 3.1 years [81].

8246 patients participated in the Vertis CV trial with ertugliflozin. The patients had diabetes and atherosclerotic cardiovascular disease. The median duration of follow-up was 3.5 years [82].

The SGLT2 inhibitors studied were non-inferior to placebo and therefore cardiovascular safe. Canagliflozin and empagliflozin were also superior to placebo. These medicines caused less cardiovascular morbidity and mortality than placebo. 


\section{Canagliflozin}

The effects of canagliflozin were investigated in the Canvas program, integrating data from two trials with over 10,000 patients with diabetes and high cardiovascular risk. The primary endpoint was death from cardiovascular causes, nonfatal myocardial infarction or nonfatal stroke. This endpoint occurred in 26.9 patients per 1,000 patient years with canagliflozin and in 31.5 patients per 1,000 patient years with placebo. The difference was statistically significant: $\mathrm{HR}=0.86 ; 95 \% \mathrm{CI}=0.75$ to 0.97 . There was no significant reduction on any individual components of the primary endpoint, any hospitalization, hospitalisation due to heart failure [79].

The NNT was 224 for 2.4 years [79].

Although the primary endpoint occurred less frequently with canagliflozin, no significant reduction of cardiovascular mortality [HR $0.87,95 \%$ CI 0.72-1.06] or death from any cause was observed [HR 0.89, 95\% CI 0.74-1.01] [79].

\section{Dapagliflozin}

The DECLARE-TIMI 53 study on dapagliflozin had 2 primary end points. The primary safety outcome was a composite of major adverse cardiovascular events [MACE], defined as cardiovascular death, non-fatal myocardial infarction, or ischemic stroke. The primary efficacy outcomes were MACE and a composite of cardiovascular death or hospitalization for heart failure. Secondary efficacy outcomes were a renal composite [ $\geq 40 \%$ decrease in estimated glomerular filtration rate to $<60 \mathrm{ml}$ per minute per $1.73 \mathrm{~m}^{2}$ of bodysurface area, new end-stage renal disease, or death from renal or cardiovascular causes] and death from any cause. Dapagliflozin showed non-inferiority compared to placebo, but superiority could not be demonstrated. This first endpoint occurred in $8.8 \%$ of patients with dapagliflozin and $9.5 \%$ of patients with placebo. The difference was not statistically significant: $\mathrm{HR}=0.93 ; 95 \% \mathrm{CI}=0.84$ to 1.03 [80].

The second primary endpoint was a combination of cardiovascular death and hospital admissions due to heart failure. This endpoint occurred in $4.9 \%$ of patients with dapagliflozin and $5.8 \%$ of patients with placebo. The difference was statistically significant: $\mathrm{HR}=0.83 ; 95 \% \mathrm{CI}=0.73$ to 0.95 . The significance was due to the effect on hospital admissions due to heart failure $2.5 \%$ vs $3.3 \%$ ]. The cardiovascular mortality was not significantly different $[2.9 \%$ in both groups] [80].
The DAPA-HF study investigated the effects of dapagliflozin 10 mg once daily and placebo in 4744 patients with New York Heart Association class II, III, or IV heart failure and an ejection fraction of $40 \%$ or less to receive either dapagliflozin [at a dose of $10 \mathrm{mg}$ once daily] or placebo, in addition to recommended therapy. The primary outcome was a composite of worsening heart failure [hospitalization or an urgent visit resulting in intravenous therapy for heart failure] or cardiovascular death.

Over a median of 18.2 months, the primary outcome occurred in $16.3 \%$ of patients [16.3\%] in the dapagliflozin group and in $21.2 \%$ ] in the placebo group [HR 0.74; 95\% CI, 0.65 to 0.85 ; $\mathrm{P}<0.001$ ].

A first worsening heart failure event occurred in $10.0 \%$ of patients in the dapagliflozin group and in $13.7 \%$ of patients in the placebo group [HR, 0.70; 95\% CI, 0.59 to 0.83]. Death from cardiovascular causes occurred in $9.6 \%$ and $11.5 \%$ of patients, respectively [HR 0.82; $95 \% \mathrm{CI}, 0.69$ to 0.98 ]; and death from any course was seen in $11.6 \%$ and $13.9 \%$ ], respectively [HR, 0.83; 95\% CI 0.71 to 0.97$]$ [83].

In an analysis of the DAPA-HF study effects on heart failure in patients with diabetes were similar to those in patients without diabetes [84].

\section{Empagliflozin}

The effects of empagliflozin on cardiovascular morbidity and mortality were investigated in the double-blind Empa-reg study. The study involved over 7,000 patients, with a median observation time of 3.1 years. The primary composite endpoint was death from cardiovascular causes, nonfatal myocardial infarction, or nonfatal stroke. The primary endpoint was observed in $10.5 \%$ of patients treated with empagliflozin and in $12.1 \%$ in the placebo group [HR 0.86, 95\% CI 0.74-0.99]. The incidence of death from cardiovascular causes and all-cause mortality was significantly lower for empagliflozin: $2.7 \%$ vs $4.1 \%$, [38\% relative risk reduction] and 5.7\% and $8.3 \%$ [32\% relative risk reduction]. The incidence of hospitalisation for heart failure was significantly lower for empagliflozin: $2.7 \%$ vs $4.1 \%, 35 \%$ relative risk reduction [81].

The NNT was 63 for 3.1 years [81].

A meta-analysis [based on the above studies] confirmed the positive effects of SGLT-2 inhibitors on cardiovascular endpoints [85]. 
One meta-analysis [including data from the above study] confirmed positive effects on MACE [86].

No positive effects on the incidence of cerebrovascular events were observed in the Empa-reg study [87]. A significant reduction of heart failure was observed for empagliflozin compared to placebo [88]. This was confirmed in another study [89].

The Emperor reduced trial assigned 3730 patients with class II-IV heart failure and an ejection fraction of $40 \%$ or less to receive empagliflozin [10 mg once daily] or placebo, in addition to recommended therapy. The primary outcome was a composite of cardiovascular death or hospitalization for worsening heart failure.

During a median of 16 months, a primary outcome event occurred in $19.4 \%$ of patients in the empagliflozin group and in $24.7 \%$ of patients in the placebo group [HR 0.75 ; $95 \%$ CI 0.65 to 0.86 ; $\mathrm{P}<0.001]$. The effect of empagliflozin on the primary outcome was consistent in patients regardless of the presence or absence of diabetes. The total number of hospitalizations for heart failure was lower in the empagliflozin group than in the placebo group [HR, 0.70; 95\% CI, 0.58 to $0.85 ; \mathrm{P}<0.001$ ] [89].

It should be noted that the cost of prevention of one serious cardiovascular event with canagliflozin or empagliflozin is high [about 80,000 euro per event] [90] and that the results are only valid in high risk patients. The cost of preventing one event in a more general diabetes type 2 population would be considerably higher than that.

\section{Ertugliflozin}

In the Vertis CV trial, patients with type 2 diabetes and atherosclerotic cardiovascular disease were randomized to receive $5 \mathrm{mg}$ or $15 \mathrm{mg}$ of ertugliflozin or placebo once daily. With the data from the two ertugliflozin dose groups pooled for analysis, the primary objective was to show the noninferiority of ertugliflozin to placebo with respect to the primary outcome, major adverse cardiovascular events [a composite of death from cardiovascular causes, nonfatal myocardial infarction, or nonfatal stroke]. The first key secondary outcome was a composite of death from cardiovascular causes or hospitalization for heart failure.

A total of 8246 patients underwent randomization and were followed for a mean of 3.5 years. Among 8238 patients who received at least one dose of ertugliflozin or placebo, a major adverse cardiovascular event occurred in $11.9 \%$ of patients in the ertugliflozin group and in $11.9 \%$ in the placebo group as well [hazard ratio, 0.97; 95.6\% confidence interval [CI], 0.85 to 1.11 ; $\mathrm{P}<0.001$ for noninferiority]. Death from cardiovascular causes or hospitalization for heart failure occurred in $8.1 \%$ in the ertugliflozin group $9.1 \%$ in the placebo group [hazard ratio, $0.88 ; 95.8 \% \mathrm{CI}, 0.75$ to 1.03 ; $\mathrm{P}=0.11$ for superiority]. The hazard ratio for death from cardiovascular causes was 0.92 [95.8\% CI, 0.77 to 1.11 ], and the hazard ratio for death from renal causes, renal replacement therapy, or doubling of the serum creatinine level was 0.81 [ $95.8 \% \mathrm{CI}, 0.63$ to 1.04]. Amputations were performed in $2.0 \%$ who received the 5 -mg dose of ertugliflozin and in $2.1 \%$ who received the 15-mg dose, as compared with 45 patients $1.6 \%$ who received placebo [82].

\section{Observational studies}

The observational CVD-Real Nordic study investigated the effects of SGLT-2 inhibitors [94\% dapagliflozin] compared SGLT-2 inhibitors with DPP-4 inhibitors regarding association with MACE, hospital events for heart failure, atrial fibrillation and severe hypoglycaemia in a real world setting in diabetes 2 patients. The incidence of all-cause mortality HR $0.44,95 \%$ CI $0.33-0.60]$, was significantly lower for SGLT-2 inhibitors. The total follow-up was over 38,000 patient years [in about a 1:3 ratio for SGLT-2 and DPP4 inhibitors]. The incidence of MACE [HR 0.79, 95\% CI 0.67-0.94] and hospital events for heart failure HR 0.62, 95\% CI 0.50-0.77] was significantly lower for SGLT-2 inhibitors. No significant differences were observed on other endpoints [91].

The same study group investigated cardiovascular morbidity and mortality of SGLT-2 inhibitors versus other glucose lowering drugs. The incidence of cardiovascular mortality HR 0.53, 95\% CI 0.40-0.71], was significantly lower for SGLT-2 inhibitors. No significant differences were observed on other morbidity endpoints. The incidence of MACE [HR 0.78, 95\% CI 0.69-0.87] and hospitalisation for heart failure HR 0.70, 95\% CI 0.61-0.81] was significantly lower for SGLT-2 inhibitors. No significant differences were observed on other morbidity endpoints [92].

\section{Effects on microvascular complications} Neuropathy

Foot problems occur frequently as a complication of diabetes, especially in the elderly. Both vascular and neurological factors 
play a role. The prevalence of diabetic neuropathy in patients over 60 years may be above $50 \%$. Diabetic neuropathy is a dreaded complication of type 2 diabetes mellitus, which can manifest itself in paresthesia, burning sensation or decreased pain sensation, especially in the feet. This can lead to ulceration, infection and gangrene or amputation of the feet [2].

The UKPDS 33 study showed no significant differences between the intensive and conventional treatment on individual endpoints related to diabetic neuropathy, such as amputation.

No specific studies using SGLT-2 inhibitors were performed.

\section{Retinopathy}

Diabetic retinopathy is the main cause of blindness. Older patients with diabetes also have an increased risk of other eye disorders, such as glaucoma, cataracts and macular degeneration. Regular eye control is very important [2].

No specific studies using SGLT-2 inhibitors were performed.

\section{Nephropathy}

The first indication of diabetic nephropathy is microalbuminuria, followed by proteinuria. Diabetic nephropathy is one of the most important indications for hemodialysis [2]. Various drugs, including angiotensin II antagonists and ACE inhibitors, may reduce renal impairment in patients with type 2 diabetes mellitus.

Nephropathy is a major complication of diabetes mellitus. About $25 \%$ of type 2 diabetes mellitus patients develop microalbuminuria within 10 years and approximately $5 \%$ of patients develop macro albuminuria. Only a relatively small proportion [0.8\%] exhibits increased serum creatinine or undergoes renal function replacement therapy. In particular, the last group showed a significant increase in mortality [93].

Canagliflozin had a more favourable effect on UACR compared to glimepiride [94]. The effects of canagliflozin were investigated in the Canvas program, integrating data from two trials with over 10,000 patients with diabetes and high cardiovascular risk. The primary endpoint was death from cardiovascular causes, nonfatal myocardial infarction or nonfatal stroke. The effects on renal events were investigated as well. Progression of albuminuria occurred significantly less frequent with canagliflozin compared to placebo: 8.94 vs 12.87 events per 100 patient years [HR 0.73, 95\% CI 0.67-0.79] [79].

The CREDENCE study on canagliflozin involved 4,401 patients with chronic kidney damage [30 to $90 \mathrm{ml} / \mathrm{min} / 1.73 \mathrm{~m} 2 \mathrm{eGFR}$ ] with severe albuminuria. The primary endpoint was a combination of end-stage renal failure, doubling of serum creatinine that persisted for longer than 30 days, and renal or cardiovascular death. Patients were followed for an average of 2.62 years [Can50]. The CREDENCE study was stopped prematurely when an interim analysis showed that canagliflozin achieved a significant result at the primary endpoint compared to placebo. The primary composite endpoint had occurred in 245 of the 2,202 patients with canagliflozin and in 340 of the 2,199 patients with placebo. This means that 22 patients must be treated with canagliflozin instead of placebo for 2.5 years to prevent 1 case of end-stage renal failure, doubling of serum creatinine, renal or cardiovascular death [95].

The DAPA-CKD study randomised 4304 participants with an estimated GFR of 25 to $75 \mathrm{ml}$ per minute per $1.73 \mathrm{~m} 2$ of body-surface area and a urinary albumin-to-creatinine ratio [with albumin measured in milligrams and creatinine measured in grams] of 200 to 5000 to dapagliflozin $10 \mathrm{mg}$ or placebo. The primary outcome was a composite of a sustained decline in the estimated GFR of at least $50 \%$, end-stage kidney disease, or death from renal or cardiovascular causes.

The study was stopped prematurely because of the observed difference in efficacy between the groups., a primary outcome event occurred in 9,2\% of participants in the dapagliflozin group and in $14.5 \%$ in the placebo group [HR 0.61; $95 \%$ CI 0.51 to $0.72 ; \mathrm{P}$ $<0.001]$, over a median of 2.4 years. The number needed to treat to prevent one primary outcome event was 19 [95\% CI, 15 to 27]]. The HR for the composite of a sustained decline in the estimated GFR of at least 50\%, ESRD, or death from renal causes was 0.56 [95\% CI, 0.45 to $0.68 ; \mathrm{P}<0.001$ ]. Death occurred in 101 participants [4.7\%] in the dapagliflozin group and 146 participants [6.8\%] in the placebo group [HR, 0.69; 95\% CI, 0.53 to $0.88 ; \mathrm{P}=0.004$ ]. The effects of dapagliflozin were similar in participants with type 2 diabetes and in those without type 2 diabetes [96]. 
Empagliflozin has demonstrated a decreased UACR compared to placebo in the large scale EMPA-REG OUTCOME study. This decrease was more pronounced in patients with existing macroalbuminuria [32\%] or microalbuminuria [25\%] than in subjects with normoalbuminuria [7\%]. The likelihood of an improvement in albuminuria was significantly greater in the empagliflozin groups compared to placebo [97].

In patients with type 2 diabetes at high cardiovascular risk, empagliflozin was associated with slower progression of kidney disease and lower rates of clinically relevant renal events than was placebo when added to standard care. Incident or worsening nephropathy occurred in $12.7 \%$ in the empagliflozin group and in $18.8 \%$ in the placebo group [hazard ratio in the empagliflozin group, $0.61 ; 95 \%$ confidence interval, 0.53 to 0.70 ; $\mathrm{P}<0.001$ ]. Doubling of the serum creatinine level occurred in $1.5 \%$ in the empagliflozin group and in $2.6 \%$ in the placebo group, a significant relative risk reduction of $44 \%$. There was no significant between-group difference in the rate of incident albuminuria [98].

The Emperor reduced trial assigned 3730 patients with class II-IV heart failure and an ejection fraction of $40 \%$ or less to receive empagliflozin [10 mg once daily] or placebo, in addition to recommended therapy. The second secondary outcome was the rate of the decline in the estimated GFR during double-blind treatment. The annual rate of decline in the estimated glomerular filtration rate was slower in the empagliflozin group than in the placebo group [-0.55 vs. $-2.28 \mathrm{ml}$ per minute per $1.73 \mathrm{~m} 2$ of body-surface area per year, $\mathrm{P}<0.001]$, and empagliflozin-treated patients had a lower risk of serious renal outcomes [89].

A recent meta-analysis including studies with empagliflozin [EMPA-REG OUTCOME], canagliflozin [CANVAS Program and CREDENCE], and dapagliflozin [DECLARE-TIMI 58]. involved 38723 participants. SGLT2 inhibitors reduced the risk of dialysis, transplantation, or death due to kidney disease [RR 0•67, 95\% CI 0.52$0 \cdot 86, p=0 \cdot 0019]$, the effect was consistent across studies. SGLT2 inhibitors also reduced end-stage kidney disease [0.65, 0.53-0.81, $\mathrm{P}<0 \cdot 0001]$, and acute kidney injury [0.75, 0.66-0.85, $\mathrm{P}<0.0001]$, with consistent benefits across studies [99].

No significant difference in renal effects was observed between ertugliflozin and placebo in the Vertis CV study, although a trend towards positive effects was observed [82].

These results should be interpreted with caution: HbA1c and blood pressure were significantly lower in the intention to treat analyses and provided no comment of per protocol analysis, which is mandatory to claim non inferiority in these trials and which has to be shown before moving to superiority analyses. It's unclear if the differences in renal disease markers would be secondary to these management differences or to the study drug itself [taking into account low adherence to assigned treatment in these studies].

\begin{tabular}{|l|c|c|c|c|c|}
\hline & $\begin{array}{c}\text { Macrovascular } \\
\text { complications }\end{array}$ & Neuropathy & Retinopathy & Nephropathy & Score \\
\hline Max score & $60 \%$ & $10 \%$ & $10 \%$ & $20 \%$ & $100 \%$ \\
\hline Canagliflozin & $36 \%$ & $5 \%$ & $5 \%$ & $20 \%$ & $66 \%$ \\
\hline Dapagliflozin & $43 \%$ & $5 \%$ & $5 \%$ & $20 \%$ & $73 \%$ \\
\hline Empagliflozin & $46 \%$ & $5 \%$ & $5 \%$ & $20 \%$ & $76 \%$ \\
\hline Ertugliflozin & $24 \%$ & $5 \%$ & $5 \%$ & $10 \%$ & $44 \%$ \\
\hline
\end{tabular}

Table i

Citation: Robert Janknegt., et al. "SGLT-2 Inhibitors: Drug Selection by Means of the System of Objectified Judgement Analysis Method". Acta Scientific Pharmaceutical Sciences 5.7 (2021): 91-112. 
This was scored as follows

\section{Discussion}

Canagliflozin, dapagliflozin, empagliflozin and ertugliflozin do not cause more cardiovascular morbidity and mortality than placebo in patients with a high cardiovascular risk. No studies are available regarding patients without high cardiovascular risk. The results of the endpoint studies cannot be compared directly, because of differences in the patient population. Some studies were performed in patients with existing cardiovascular disease, whereas other studies also included patients at high risk of developing cardiovascular disease, but without existing disease.

Canagliflozin, dapagliflozin and empagliflozin cause less cardiovascular morbidity and mortality than placebo in patients with a high cardiovascular risk. This has not been demonstrated for ertugliflozin. For empagliflozin, 63 people must be treated for 3.1 years to prevent 1 case of cardiovascular death, non-fatal myocardial infarction or non-fatal stroke. For canagliflozin this is 224 patients for 2.4 years. This was not established for dapagliflozin, because significance was not reached in the DECLARE-TIMI 53 study, which also included patients without existing cardiovascular disease.

Canagliflozin and empagliflozin are awarded 36\% [60\% of the available $60 \%$ for this sub criterion].

Dapagliflozin is awarded 33\% [55\% of the available $60 \%$ for this sub criterion], because one of the primary endpoints was not reached [in a different patient population].

Ertugliflozin is awarded $24 \%$ [ $40 \%$ of the available $60 \%$ for this sub criterion].

Dapagliflozin and empagliflozin are awarded 10\% extra because of the documented effects in patients with heart failure: $43 \%$ and $46 \%$, respectively for CV effects.

Safety

Fractures

Canagliflozin showed a decrease in BMD and an increased incidence of fractures $[100,101]$, whereas no effect on the incidence of fractures was seen in dapagliflozin or empagliflozin [102-108].

Several meta-analyses have studied the relationship between
SGLT-2 inhibitors as a group and fractures. None of these studies found a significant increase in fractures compared to placebo or other oral antidiabetics [109-111]. Most studies were however of short duration.

\section{Hypoglycaemia}

The incidence of hypoglycaemia during monotherapy with SGLT-2 inhibitors is low. An increased risk of hypoglycaemia compared to placebo was found when these drugs are combined with insulin or sulfonylureas [95-108,112-114]. A pooled analysis of studies with empaglifozin did not show an increased incidence of hypoglycaemia in combination with insulin or sulfonylureas [115].

\section{Volume depletion}

The incidence of all adverse reactions related to volume depletion [such as postural dizziness, orthostatic hypotension, hypotension, dehydration, and syncope] was similar for SGLT-2 inhibitor and for placebo or other oral antidiabetics. The incidence was increased in patients with high risk, such as a history of cardiovascular disease, impaired renal function or age $>75$ years [10$108,116,117]$.

\section{Genital mycotic infections}

Vulvovaginal candidiasis and balanitis are observed 3 times more frequently during use of SGLT-2 inhibitors than with placebo. Between $0.5 \%$ and $1 \%$ of patients discontinued treatment because of these complaints [105-108,112,113,117,118].

\section{Amputation}

The FDA has issued warnings of increased leg and foot amputation risk be added to canagliflozin drug labelling. The risk associated with other SGLT2 inhibitors was uncertain. One meta-analysis investigated the risk of amputation in 14 clinical trials. SGLT2 inhibitors as a group were not associated with an increased risk of diabetic foot syndrome compared with placebo: OR 1.05, 95\% CI: 0.58-1.89. SGLT-2 inhibitors as a class were not significantly associated with amputation risk [OR 1.40, 95\% CI:0.81-2.41], but canagliflozin showed an increased incidence of amputation in participants using canagliflozin [OR 1.89, 95\% CI: 1.37-2.60], compared with oral antidiabetics or placebo. Empagliflozin did not show an increased incidence of amputation [119].

In ongoing, long-term clinical studies of canagliflozin in type 2 
diabetes patients with cardiovascular disease [CVD] or at high risk for CVD, an increase in cases of lower limb amputation [primarily of the toe] has been observed in patients treated with canagliflozin [SPC Invokana]. This was confirmed in the Canvas study: 0.63 versus 0.34 amputations per 100 patient years [79]. As an underlying mechanism has not been established, risk factors, apart from general risk factors for amputation are unknown [SPC Invokana]. It is unclear whether this applies to the other SGLT-2 inhibitors as well [SPC Forxiga, Jardiance]. The large scale EMPA-REG study did not present data indicating an increased risk for empagliflozin.

A large US database study, investigating 142800 new users of canagliflozin, 110897 new users of other SGLT- 2 inhibitors and 460885 new users of non-SGLT-2 antidiabetics], showed no increased risk of amputation for canagliflozin. The estimate for lower limb amputation with canagliflozin vs non-SGLT- 2 antidiabetics was 0.75 [95\% CI, 0.40-1.41] in the on-treatment analysis and 1.01 [95\% CI, 0.93-1.10] in the intent-to-treat analysis [120].

\section{Increased creatinine}

An increase in serum creatinine has been found for dapagliflozin [SPC Forxiga]. The Canvas trial showed a possible positive effect of canagliflozin in the progression of albuminuria [HR 0.73; IC95\% 0.67- 0.79] and in a composite outcome [glomerular filtration rate, the need for renal transplant and renal related death; HR 0.60; IC95\% 0.47-0.77] [79].

\section{Cancer risk}

The available evidence from short-term RCTs did not indicate a significantly increased risk of overall cancer among individuals with type 2 diabetes using SGLT2 inhibitors. Most studies were however of short duration [121].

\section{Infections}

A meta-analysis showed that SGLT-2 inhibitors increased the risk of genital infections compared to placebo [RR 3.37, 95\% CI 2.89-3.93] and active comparator [RR 3.89, 95\% CI 3.14-4.82]. The risk of urinary tract infection was not increased with SGLT-2 inhibitors as a group, compared to placebo or active comparators. Dapagliflozin $10 \mathrm{mg}$ daily was associated with a significantly increased risk of UTI compared to placebo [RR 1.33, 95\% CI 1.10-1.61] [122].

One meta-analysis showed that canagliflozin, dapagliflozin and empagliflozin were associated with a higher risk of genital infections than placebo, with ORs ranging from 3.21 [95\% CI 2.08-4.93] for dapagliflozin $2.5 \mathrm{mg}$ to 5.23 [95\% CI 3.86-7.09] for canagliflozin $300 \mathrm{mg}$ [123].

\section{Other adverse events}

Fournier's gangrene

Post-marketing cases of necrotising fasciitis of the perineum, [also known as Fournier's gangrene], have been reported in female and male patients taking SGLT- 2 inhibitors. This is a rare but serious and potentially life-threatening event that requires urgent surgical intervention and antibiotic treatment. Patients should be advised to seek medical attention if they experience a combination of symptoms of pain, tenderness, erythema, or swelling in the genital or perineal area, with fever or malaise. Be aware that either uro-genital infection or perineal abscess may precede necrotising fasciitis. If Fournier's 6 gangrene is suspected, all SGLT-2 inhibitors should be discontinued and prompt treatment [including antibiotics and surgical debridement] should be instituted] [105-108].

\section{Ketoacidosis}

Rare cases of diabetic ketoacidosis [DKA], including life-threatening and fatal cases, have been reported in clinical trials and postmarketing in patients treated with SGLT- 2 inhibitors, and cases have been reported in clinical trials with the SGLT- 2 inhibitors. In a number of cases, the presentation of the condition was atypical with only moderately increased blood glucose values, below 14 $\mathrm{mmol} / \mathrm{l}$ [250 mg/dl]. It is not known if DKA is more likely to occur with higher doses of SGLT- 2 inhibitors. Patients should be assessed for ketoacidosis immediately if these symptoms occur, regardless of blood glucose level. In patients where DKA is suspected or diagnosed, treatment with SGLT-2 inhibitors should be discontinued immediately [105-108].

Treatment should be interrupted in patients who are hospitalised for major surgical procedures or acute serious medical illnesses. Monitoring of ketones is recommended in these patients. Measurement of blood ketone levels is preferred to urine. Treatment with SGLT-2 inhibitors may be restarted when the ketone values are normal and the patient's condition has stabilised. Before initiating therapy with SGLT-2 inhibitors, factors in the patient history that may predispose to ketoacidosis should be considered. Patients who may be at higher risk of DKA include patients with a low beta-cell function reserve [e.g. type 2 diabetes patients with low C-peptide or latent autoimmune diabetes in adults [LADA] or patients with a history of pancreatitis], patients with conditions 
that lead to restricted food intake or severe dehydration, patients for whom insulin doses are reduced and patients with increased insulin requirements due to acute medical illness, surgery or alcohol abuse. SGLT2 inhibitors should be used with caution in these patients. Restarting SGLT2 inhibitor treatment in patients with previous DKA while on SGLT-2 inhibitor treatment is not recommended, unless another clear precipitating factor is identified and resolved [105-108].

Ketoacidosis and Fournier's gangrene are rarely observed during treatment with SGLT-2 inhibitors. There are no indications of relevant differences between the medicines in this effect.

Dapagliflozin, empagliflozin and ertugliflozin are awarded $70 \%$ for safety.

The incidence of amputations may be higher for canagliflozin. This medicine is awarded $60 \%$.

Tolerability

Frequent, non-serious adverse effects

SGLT-2 inhibitors are also usually well tolerated, with an incidence of adverse events comparable to placebo [116-123], with the exception of a higher incidence of genital infections [124].

One meta-analysis included studies comparing canagliflozin $100 \mathrm{mg}$ and $300 \mathrm{mg}$ to sitagliptin. No differences were found in the incidence of adverse events, serious adverse events, discontinuation, urinary tract infections and hypoglycaemia. The only significant difference between both medicines was the higher incidence of genital mycotic infections, which was higher for canaglifozin 100 mg [RR 4.32]. The risk was significantly increased in both men and women [125].

Canagliflozin showed a higher incidence of osmotic diuresis related adverse events compared to placebo in a meta-analysis of monotherapy [Meta 35] and a higher incidence of pollakiuria in combination with other oral antidiabetics compared with placebo [126].

Empagliflozin showed a higher incidence of genital infections compared to placebo in a meta-analysis of 15 randomised studies [127].

All SGLT-2 inhibitors are awarded 70\%.

\section{Drug interactions}

Few clinically relevant interactions have been found for SGLT-2 inhibitors.

Pharmacodynamic interactions may occur with diuretics, resulting in additional volume depletion and hypotension.

Rifampicin decreases the AUC of SGLT-2 inhibitors: 51\% reduction for canagliflozin, 22\% for dapagliflozin, 35\% for empagliflozin and 39\% for ertugliflozin [105-108,127].

Clinically insignificant increases in the AUC of digoxin and simvastatin [less than 20\%] have been described in combination with SGLT-2 inhibitors [105-108].

All SGLT-2 inhibitors are awarded 75\%.

\section{Documentation}

The documentation is summarized below. The documentation included only double-blind studies with at least 25 patients per treatment arm and a study duration of at least 4 weeks.

\section{SOJA score}

The SOJA score is presented in the Table below.

\begin{tabular}{|l|c|c|c|c|c|}
\hline & Studies & Patients & Years & $\begin{array}{c}\text { Patient } \\
\text { years } \\
\text { (million) }\end{array}$ & Score \\
\hline Canagliflozin & $>20$ & $>1000$ & 8 & $>100$ & $95 \%$ \\
\hline Dapagliflozin & $>20$ & $>1000$ & 9 & $>100$ & $97 \%$ \\
\hline Empagliflozin & $>20$ & $>1000$ & 7 & $>100$ & $92 \%$ \\
\hline Ertugliflozin & 7 & $>1000$ & 4 & $>100$ & $68 \%$ \\
\hline
\end{tabular}

Table j: Overview of documentation

\section{Applied methodology}

Drug selection was performed by means of the SOJA method, which is a well-established rational and transparent way of selecting medicines [or in this case inhalation devices] within a therapeutic class from a formulary perspective. The evaluation of the criteria in the SOJA method is highly standardized in order to promote unbiased judgement of drugs from various pharmacological categories based on clinically relevant criteria. Of course, there is potential debate on the correct scoring system with respect to 


\begin{tabular}{|l|c|c|c|c|c|}
\hline & Weight & Canagliflozin & Dapagliflozin & Empagliflozin & Ertugliflozin \\
\hline Ease of use & 50 & 50 & 45 & 45 & 45 \\
\hline Available dosage forms & 50 & 30 & 30 & 30 & 40 \\
\hline Efficacy & 200 & 138 & 138 & 138 & 138 \\
\hline $\begin{array}{l}\text { Documented effects on clinically } \\
\text { relevant endpoints }\end{array}$ & 250 & 165 & 182 & 190 \\
\hline Safety & 200 & 120 & 140 & 140 & 140 \\
\hline Tolerability & 100 & 140 & 140 & 140 & 140 \\
\hline Interactions & 50 & 38 & 38 & 38 & 38 \\
\hline Documentation & 100 & 95 & 97 & 813 & 68 \\
\hline Total & 1000 & 776 & 810 & & 719 \\
\hline
\end{tabular}

\section{Table k}

each criterion and individual decisions are highly subjective. This is the case with any method used to quantify properties of drugs. The SOJA method is intended as a tool for rational drug decision making, enabling clinicians and pharmacists to include all relevant aspects of a certain group of drugs, thereby preventing formulary decisions being based on only one or two criteria. Besides this, possible "hidden criteria" [such as personal financial interest] are excluded from the decision making process. The outcome of this study should be seen as the basis for discussions within formulary committees and not as the absolute truth.

\section{Outcomes}

Relatively limited differences in score are seen between the SGLT-2 inhibitors [about $12 \%$ between the highest and lowest score]. Of course, the present scoring is based on the weights assigned by the authors. The essence of the SOJA method is that users of the method may assign their own relative weight to each selection criterion. This interactive program is available on the internet: www.tablet.sojaonline.nl. Other relative weights will of course affect the relative scores for the medicines.

It should be stressed that one single SGLT-2 inhibitor may not be suitable for all patients. All SGLT-2 inhibitors have minor differences regarding advantages and disadvantages, but these may be more pronounced in individual patients.

The relatively high scores for empagliflozin and dapagliflozin are caused by a favourable score for the criteria documented ef- fects on clinical endpoints [including effects on heart failure] and safety. Canaglilozin also scores well, but this drug has a slightly lower score for safety and no studies on effects on heart failure are available.

Strength and limitations of the methodology Selection criteria

Of course, other selection criteria could be applied as well. We did not include Contra-indications and Warnings and Precautions in the matrix. There were no relevant differences between the SGLT-2 antagonists in this respect. Differences in the incidence of bleeding or drug interactions were accounted for in the current selection criteria.

Variability of the AUC is a standard criterion for SOJA. Its relevance for SGLT-2 antagonists is unclear. That is why a low weight was assigned to this criterion. When one considers this criterion to be completely irrelevant, a zero weight can be given to this criterion in the interactive program.

Clinical efficacy and safety are the most important selection criteria for all groups of medicines. Unfortunately these criteria are difficult to score for SGLT-2 inhibitors because of the lack of direct comparative studies and differences in patient populations, study design and applied endpoints. Meta-analyses and registry data may be of value in the judgement of efficacy and safety. All data sources have specific strengths and weaknesses.

Acquisition cost was not included as a selection criterion to 
make the score internationally applicable. The present matrix can be used as a pre-selection tool of the most suitable SGLT-2 inhibitors from a quality point of view. Because prices may different institutions and in different healthcare systems, individual procurement procedures should lead to a selection of the best options.

\section{Judgement of properties of SGLT-2 inhibitors}

Double-blind comparative studies are the most important source of information of the determination of clinical efficacy and tolerability. These studies usually have limitations in the selection of patients and a limited duration of the study. No direct comparative studies are available, which makes it possible to reliably evaluate the SGLT-2 inhibitors on the most important selection criteria, clinical efficacy, documented effects on clinically relevant endpoints and safety. This score should therefore be considered as preliminary. On the other hand, it seems quite unlikely that large scale direct comparative studies with more than 2 SGLT- 2 inhibitors will be published in the near future, so we will have to deal with indirect comparisons. An analysis of the landmark studies with SGLT-2 inhibitors showed a positive effect on mortality for empagliflozin in patients with existing atherosclerotic cardiovascular disease [HR 0.62, 95\% CI 0.49-0.77], whereas this was not documented for the other SGLT-2 inhibitors [128].

Because of the lack of direct comparative studies, the results of meta-analyses and registries were also taken into consideration. These kinds of studies also have limitations. The quality of metaanalyses is as good as the quality of the studies which are included. Patient populations may be quite different for patients treated with the individual SGLT-2 inhibitors in registry studies.

Formulary choices versus decisions in treatment of individual patients

It should be stated that formulary selection is a different process than decision making in individual patients. Selection criteria like variability in AUC, number of approved indications and documentation are typical criteria that may be relevant from a formulary perspective, but not for the selection of an SGLT-2 inhibitor in individual patients.

The above described differences in properties of SGLT-2 inhibitors may lead to drug and dosage choices based on the specific situation of the patient, such as comedication [risk of interactions], comorbidity, renal function impairment and individual tolerability.

\section{Conclusions}

We found limited differences in the scores of the available SGLT2 inhibitors. Empagliflozin and dapagliflozin showed the highest

\section{Bibliography}

1. Lebovitz HE. "Oral therapies for diabetic hyperglycemia”. Endocrinology and Metabolism Clinics of North America 30 (2001): 909-933.

2. Rosenstock J. "Management of type 2 diabetes mellitus in the elderly: special considerations”. Drugs Aging 18.1 (2001): 3144.

3. Davis TM., et al. "Risk factors for stroke in type 2 diabetes mellitus: United Kingdom Prospective Diabetes Study (UKPDS) 29”. Archives of Internal Medicine 159 (1999): 1097-1103.

4. Pratley RE., et al. "Rapid acting insulinotropic agents: restoration of early insulin secretion as a physiologic approach to improve glucose control". Current Pharmaceutical Design 7 (2001): 1375-1397.

5. Bailey CJ and Turner RC. "Metformin". The New England Journal of Medicine 334 (1996): 574-579.

6. Dunn CJ and Peters DH. "Metformin. A review of its pharmacological properties and therapeutic use in non-insulin-dependent diabetes mellitus". Drugs 49 (1995): 721-749.

7. Mehnert H. "Metformin, the rebirth of a biguanide: mechanism of action and place in the prevention and treatment of insulin resistance". Experimental and Clinical Endocrinology and Diabetes 109 (2001): S259-264.

8. Palmer KJ and Brogden RN. "Gliclazide. An update of its pharmacological properties and therapeutic efficacy in non-insulindependent diabetes mellitus". Drugs 46 (1993): 92-125.

9. Muller G., et al. "Extrapancreatic effects of sulfonylureas--a comparison between glimepiride and conventional sulfonylureas". Diabetes Research and Clinical Practice 28 (1995): S115137. 
10. Langtry HD., et al. "Glimepiride. A review of its use in the management of type 2 diabetes mellitus". Drugs 55 (1998): 563584.

11. Davies MJ. "Insulin secretagogues". Current Medical Research and Opinion 18 (2002): s22-30.

12. Diamant M and Heine RJ. "Thiazolidinediones in type 2 diabetes mellitus”. Current Clinical Evidence Drugs 63 (2003): 13731405.

13. Janknegt R and Steenhoek A. "The System of Objectified Judgement Analysis. A tool in rational drug selection for formulary purposes". Drugs 53 (1997): 550-562.

14. Lee JD., et al. "Recalculation of cardiovascular risk score as a surrogate marker of change in clinical care of diabetes patients: the Alphabet POEM project (Practice Of Evidence-based Medicine)". Current Medical Research and Opinion 20 (2004): 765772 .

15. UK Prospective Diabetes Study (UKPDS) Group. "Intensive blood-glucose control with sulphonylureas or insulin compared with conventional treatment and risk of complications in patients with type 2 diabetes (UKPDS 33)". Lancet 352 (1998): 837-853.

16. Manley S. "Haemoglobin A1c--a marker for complications of type 2 diabetes: the experience from the UK Prospective Diabetes Study (UKPDS)". Clinical Chemistry and Laboratory Medicine 41 (2003): 1182-1190.

17. Stratton IM., et al. "Association of glycaemia with macrovascular and microvascular complications of type 2 diabetes (UKPDS 35): prospective observational study". BMJ 321 (2000): 405412 .

18. Adler AI., et al. "Hyperglycemia and hyperinsulinemia at diagnosis of diabetes and their association with subsequent cardiovascular disease in the United Kingdom prospective diabetes study (UKPDS 47)”. American Heart Journal 138 (1999): S353359.

19. Stevens RJ., et al. "Risk factors for myocardial infarction case fatality and stroke case fatality in type 2 diabetes: UKPDS 66". Diabetes Care 27 (2004): 201-207.
20. McCulloch DK. "Treatment of blood glucose in type 2 diabetes mellitus". American Family Physician 79.1 (2009): 29-36.

21. Goldstein BJ. "Differentiating members of the thiazolidinedione class: a focus on efficacy". Diabetes/Metabolism Research and Reviews 18 (2002): S16-22.

22. Goldstein BJ., et al. "Are the metabolic effects of rosiglitazone influenced by baseline glycaemic control?". Current Medical Research and Opinion 19.3 (2003): 192-199.

23. Flier JS and Mantzoros C. "Insulin resistance: definition and clinical spectrum".

24. Scheen AJ. "Management of the metabolic syndrome". Minerva Endocrinology 29 (2004): 31-45.

25. Ovalle F and Bell DS. "Effect of rosiglitazone versus insulin on the pancreatic beta-cell function of subjects with type 2 diabetes". Diabetes Care 27 (2004): 2585-2589.

26. Smith SA., et al. "Rosiglitazone, but not glyburide, reduces circulating proinsulin and the proinsulin:insulin ratio in type 2 diabetes". The Journal of Clinical Endocrinology and Metabolism 89 (2004): 6048-6053.

27. Mauvais-Jarvis F., et al. "Therapeutic perspectives for type 2 diabetes mellitus: molecular and clinical insights". Diabetes $\mathrm{Me}$ tabolism 27 (2001): 415-423.

28. Storgaard H., et al. "Benefits and Harms of Sodium-Glucose CoTransporter 2 Inhibitors in Patients with Type 2 Diabetes: A Systematic Review and Meta-Analysis". PLoS One 11 (2016): e0166125.

29. Shyangdan DS., et al. "SGLT-2 receptor inhibitors for treating patients with type 2 diabetes mellitus: a systematic review and network meta-analysis". BMJ Open 6 (2016): e009417.

30. McNeill AM., et al. "Ertugliflozin Compared to Other Anti-hyperglycemic Agents asMonotherapy and Add-on Therapy in Type 2 Diabetes: A Systematic Literature Review and Network Meta Analysis". Diabetes Therapy 10.2 (2019): 473-491.

31. Zhang YJ., et al. "Efficacy and safety of empagliflozin for type 2 diabetes mellitus: Meta-analysis of randomized controlled trials". Medicine (Baltimore) 97 (2018): e12843. 
32. Zhong X., et al. "Efficacy and safety of empagliflozin as addon to metformin for type 2 diabetes: a systematic review and meta-analysis". European Journal of Clinical Pharmacology 72 (2016): 655-663.

33. Mearns ES., et al. "Efficacy and safety of antihyperglycaemic drug regimens added to metformin and sulphonylurea therapy in Type 2 diabetes: a network meta-analysis". Diabetic Medicine 32 (2015): 1530-1540.

34. Mearns ES., et al. "Comparative efficacy and safety of antidiabetic drug regimens added to metformin monotherapy in patients with type 2 diabetes: a network meta-analysis". PLoS One 10 (2015): e0125879.

35. Mudaliar S., et al. "Changes in insulin sensitivity and insulin secretion with the sodium glucose cotransporter 2 inhibitor dapagliflozin". Diabetes Technology and Therapeutics 16 (2014): 137-144.

36. Merovci A., et al. "Effect of Dapagliflozin With and Without Acipimox on Insulin Sensitivity and Insulin Secretion in T2DM Males". The Journal of Clinical Endocrinology and Metabolism 101 (2016): 1249-1256.

37. Leiter LA., et al. "Canagliflozin provides durable glycemic improvements and body weight reduction over 104 weeks versus glimepiride in patients with type 2 diabetes on metformin: a randomized, double-blind, phase 3 study". Diabetes Care 38 (2015): 355-364.

38. Traina S., et al. "The impact of weight loss on weight-related quality of life and health satisfaction: results from a trial comparing canagliflozin with sitagliptin in triple therapy among people with type 2 diabetes". Postgraduate Medicine 126.3 (2014): 7-15.

39. Wilding J., et al. "Dapagliflozin therapy for type 2 diabetes in primary care: Changes in HbA1c, weight and blood pressure over 2 years follow-up". Primary Care Diabetes (2017): pii:S1751-9918 (17)30072-4.

40. Bolinder J., et al. "Effects of dapagliflozin on body weight, total fat mass, and regional adipose tissue distribution in patients with type 2 diabetes mellitus with inadequate glycemic control on metformin". The Journal of Clinical Endocrinology and Me- tabolism 97 (2012): 1020-1031.

41. Rosenstock J., et al. "Effects of dapagliflozin, an SGLT2 inhibitor, on $\mathrm{HbA}(1 \mathrm{c})$, body weight, and hypoglycemia risk in patients with type 2 diabetes inadequately controlled on pioglitazone monotherapy". Diabetes Care 35 (2012): 1473-1478.

42. Bolinder J., et al. "Dapagliflozin maintains glycaemic control while reducing weight and body fat mass over 2 years in patients with type 2 diabetes mellitus inadequately controlled on metformin". Diabetes, Obesity and Metabolism 16 (2014): 159169.

43. Matthaei S., et al. "Dapagliflozin improves glycemic control and reduces body weight as add-on therapy to metformin plus sulfonylurea: a 24-week randomized, double-blind clinical trial". Diabetes Care 38 (2015): 365-372.

44. Sjöström CD., et al. "Dapagliflozin-induced weight loss affects 24-week glycated haemoglobin and blood pressure levels". Diabetes, Obesity and Metabolism 17 (2015): 809-812.

45. Wilding J., et al. “Glycated Hemoglobin, Body Weight and Blood Pressure in Type 2 Diabetes Patients Initiating Dapagliflozin Treatment in Primary Care: A Retrospective Study". Diabetes Therapy 7 (2016): 695-711.

46. Scheerer MF., et al. "Changes in HbA1c, body weight, and systolic blood pressure in type 2 diabetes patients initiating dapagliflozin therapy: a primary care database study". Diabetes Metabolism 9 (2016): 337-345.

47. Kostev K., et al. "Changes in Glycemic Control and Body Weight After Initiation of Dapagliflozin or Basal Insulin Supported Oral Therapy in Type 2 Diabetes: A Primary Care Database Study". Journal of Diabetes Science and Technology 11 (2017): 590-596.

48. Kovacs CS., et al. "Empagliflozin improves glycaemic and weight control as add-on therapy to pioglitazone or pioglitazone plus metformin in patients with type 2 diabetes: a 24-week, randomized, placebo-controlled trial”. Diabetes, Obesity and Metabolism 16 (2014): 147-158.

49. Rosenstock J., et al. "Improved glucose control with weight loss, lower insulin doses, and no increased hypoglycemia with empagliflozin added to titrated multiple daily injections of insu- 
lin in obese inadequately controlled type 2 diabetes". Diabetes Care 37 (2014): 1815-1823.

50. Neeland IJ., et al. "Empagliflozin reduces body weight and indices of adipose distribution in patients with type 2 diabetes mellitus". Diabetes and Vascular Disease Research 13 (2016): 119-126.

51. Shiba T., et al. "Efficacy and safety of empagliflozin in Japanese patients with type 2 diabetes mellitus: A sub-analysis by body mass index and age of pooled data from three clinical trials". Diabetes Research and Clinical Practice 131 (2017): 169-178.

52. Camacho P., et al. "Beneficial and detrimental effects of intensive glycaemic control, with emphasis on type 2 diabetes mellitus". Drugs Aging 17 (2000): 463-476.

53. Holman R. "The UKPDS: implications for the dyslipidaemic patient". Acta Diabetology 38 (2001): S9-14.

54. Adler AI., et al. "UKPDS 59: hyperglycemia and other potentially modifiable risk factors for peripheral vascular disease in type 2 diabetes". Diabetes Care 25 (2002): 894-899.

55. Song SH and Brown PM. "Coronary heart disease risk assessment in diabetes mellitus: comparison of UKPDS risk engine with Framingham risk assessment function and its clinical implications". Diabetic Medicine 21 (2004): 238-245.

56. Inagaki N., et al. "Effects of Baseline Blood Pressure and LowDensity Lipoprotein Cholesterol on Safety and Efficacy of Canagliflozin in Japanese Patients with Type 2 Diabetes Mellitus". Advances in Therapy 32 (2015): 1085-1103.

57. Bays HE., et al. "Dapagliflozin in patients with type II diabetes mellitus, with and without elevated triglyceride and reduced high-density lipoprotein cholesterol levels". Journal of Clinical Lipidology 11 (2017): 450-458.e1.

58. Fadini GP., et al. "Effects of the SGLT2 inhibitor dapagliflozin on HDL cholesterol, particle size, and cholesterol efflux capacity in patients with type 2 diabetes: a randomized placebo-controlled trial". Cardiovascular Diabetology 16 (2017): 42.

59. Hayashi T., et al. "Dapagliflozin decreases small dense low-density lipoprotein-cholesterol and increases high-density lipoprotein 2-cholesterol in patients with type 2 diabetes: comparison with sitagliptin". Cardiovascular Diabetology 16 (2017): 8.
60. Briand F., et al. "Empagliflozin, via Switching Metabolism Toward Lipid Utilization, Moderately Increases LDL Cholesterol Levels Through Reduced LDL Catabolism". Diabetes 65 (2016): 2032-2038.

61. Weir MR., et al. "Effect of canagliflozin on blood pressure and adverse events related to osmotic diuresis and reduced intravascular volume in patients with type 2 diabetes mellitus". Journal of Clinical Hypertension (Greenwich) 16 (2014): 875882.

62. Cefalu WT., et al. "Effects of canagliflozin on body weight and relationship to $\mathrm{HbA1c}$ and blood pressure changes in patients with type 2 diabetes". Diabetologia 58 (2015): 1183-1187.

63. Guthrie RM. "Practice pearl: the effect of canagliflozin on HbA1c and blood pressure in patients with type 2 diabetes mellitus". Postgraduate Medicine 127 (2016): 783-785.

64. Lefebvre P., et al. "Real-world glycemic, blood pressure, and weight control in patients with type 2 diabetes mellitus treated with canagliflozin-an electronic health-record-based study". Current Medical Research and Opinion 32 (2016): 1151-1159.

65. Townsend RR., et al. "Reductions in Mean 24-Hour Ambulatory Blood Pressure After 6-Week Treatment With Canagliflozin in Patients With Type 2 Diabetes Mellitus and Hypertension". Journal of Clinical Hypertension (Greenwich) 18 (2016): 43-52.

66. Pfeifer M., et al. "Effects of canagliflozin, a sodium glucose cotransporter 2 inhibitor, on blood pressure and markers of arterial stiffness in patients with type 2 diabetes mellitus: a post hoc analysis". Cardiovascular Diabetology 16 (2017): 29.

67. Kohan DE., et al. "Long-term study of patients with type 2 diabetes and moderate renal impairment shows that dapagliflozin reduces weight and blood pressure but does not improve glycemic control". Kidney International 85 (2014): 962-971.

68. Sjöström CD., et al. "Dapagliflozin-induced weight loss affects 24-week glycated haemoglobin and blood pressure levels". Diabetes, Obesity and Metabolism 17 (2015): 809-812.

69. Sjöström CD., et al. "Dapagliflozin lowers blood pressure in hypertensive and non-hypertensive patients with type 2 diabetes". Diabetes and Vascular Disease Research 12 (2015): 352358.

Citation: Robert Janknegt., et al. "SGLT-2 Inhibitors: Drug Selection by Means of the System of Objectified Judgement Analysis Method". Acta Scientific Pharmaceutical Sciences 5.7 (2021): 91-112. 
70. Weber MA., et al. "Blood pressure and glycaemic effects of dapagliflozin versus placebo in patients with type 2 diabetes on combination antihypertensive therapy: a randomised, double-blind, placebo-controlled, phase 3 study". Lancet Diabetes and Endocrinology 4 (2016): 211-220.

71. Weber MA., et al. "Effects of dapagliflozin on blood pressure in hypertensive diabetic patients on renin-angiotensin system blockade". Blood Press 25 (2016): 93-103.

72. Tikkanen I., et al. "Empagliflozin reduces blood pressure in patients with type 2 diabetes and hypertension". Diabetes Care 38 (2015): 420-428.

73. Chilton R., et al. "Effects of empagliflozin on blood pressure and markers of arterial stiffness and vascular resistance in patients with type 2 diabetes". Diabetes, Obesity and Metabolism 17 (2015): 1180-1193.

74. Mancia G., et al. "Impact of Empagliflozin on Blood Pressure in Patients With Type 2 Diabetes Mellitus and Hypertension by Background Antihypertensive Medication". Hypertension 68 (2016): 1355-1364.

75. Rosenstock J., et al. "Effect of ertugliflozin on glucose control, body weight, blood pressure and bone density in type 2 diabetes mellitus inadequately controlled on metformin monotherapy (VERTIS MET)". Diabetes, Obesity and Metabolism 20.3 (2018): 520-529.

76. Amin NB., et al. "Blood pressure-lowering effect of the sodium glucose co-transporter-2 inhibitor ertugliflozin, assessed via ambulatory blood pressure monitoring in patients with type 2 diabetes and hypertension". Diabetes, Obesity and Metabolism 17.8 (2015): 805-808.

77. Booth GL., et al. "Relation between age and cardiovascular disease in men and women with diabetes compared with non-diabetic people: a population-based retrospective cohort study". Lancet 368 (2006): 29-36.

78. Kelly TN., et al. "Systematic review: Glucose control and cardiovascular disease in type 2 diabetes". Annals of Internal Medicine 151 (2009): 394-403.

79. Neal B., et al. "Canagliflozin and Cardiovascular and Renal Events in Type 2 Diabetes". The New England Journal of Medi- cine 377 (2017): 644-657.

80. Wiviott SD., et al. "Dapagliflozin and Cardiovascular Outcomes in Type 2 Diabetes". The New England Journal of Medicine 380.4 (2019): 347-357.

81. Zinman B., et al. "Empagliflozin, Cardiovascular Outcomes, and Mortality in Type 2 Diabetes". The New England Journal of Medicine 373 (2015): 2117-2128.

82. Cannon CP., et al. "Cardiovascular Outcomes with Ertugliflozin in Type 2 Diabetes". The New England Journal of Medicine (2020).

83. McMurray JJV., et al. "Dapagliflozin in Patients with Heart Failure and Reduced Ejection Fraction". The New England Journal of Medicine 381 (2019): 1995-2008.

84. Petrie MC., et al. "Effect of Dapagliflozin on Worsening Heart Failure and Cardiovascular Death in Patients With Heart Failure With and Without Diabetes". JAMA 323 (2020): 1353-1368.

85. Lee G., et al. "Comparative effectiveness of oral antidiabetic drugs in preventing cardiovascular mortality and morbidity: A network meta-analysis". PLoS One 12 (2017): e0177646.

86. Salsali A., et al. "Cardiovascular safety of empagliflozin in patients with type 2 diabetes: a meta-analysis of data from randomized placebo-controlled trials". Diabetes, Obesity and Metabolism 18 (2016): 1034-1040.

87. Zinman B., et al. "Empagliflozin and Cerebrovascular Events in Patients With Type 2 Diabetes Mellitus at High Cardiovascular Risk". Stroke 48 (2017): 1218-1225.

88. Fitchett D., et al. "Heart failure outcomes with empagliflozin in patients with type 2 diabetes at high cardiovascular risk: results of the EMPA-REG OUTCOME® trial". European Heart Journal 37 (2016): 1526-1534.

89. Packer M., et al. "Cardiovascular and Renal Outcomes with Empagliflozin in Heart Failure". The New England Journal of Medicine 383 (2020): 1413-1424.

90. Naci H., et al. "Preventing cardiovascular events with empagliflozin: at what cost?". Lancet Diabetes and Endocrinology 3 (2015): 931. 
91. Packer M., et al. "Cardiovascular and Renal Outcomes with Empagliflozin in Heart Failure.Lower Risk of Cardiovascular Events and All-cause Mortality in Type 2 Diabetes Patients (CVD-REAL Nordic): a multinational observational study". Diabetes, Obesity and Metabolism (2017).

92. Birkeland KI., et al. "Cardiovascular mortality and morbidity in patients with type 2 diabetes following initiation of sodium-glucose co-transporter-2 inhibitors versus other glucoselowering drugs (CVD-REAL Nordic): a multinational observational analysis". Lancet Diabetes and Endocrinology (2017): pii: S2213-8587 (17)30258-9.

93. Adler AI., et al. "Development and progression of nephropathy in type 2 diabetes: the United Kingdom Prospective Diabetes Study (UKPDS 64)". Kidney International 63 (2003): 225-232.

94. Heerspink HJ., et al. "Canagliflozin Slows Progression of Renal Function Decline Independently of Glycemic Effects". Journal of the American Society of Nephrology 28 (2017): 368-375.

95. Perkovic V., et al. "Canagliflozin and Renal Outcomes in Type 2 Diabetes and Nephropathy". The New England Journal of Medicine 380 (2019): 2295-2306.

96. Heerspink HJL., et al. "Dapagliflozin in Patients with Chronic Kidney Disease". The New England Journal of Medicine 383 (2020): 1436-1446.

97. Cherney DZI., et al. "Effects of empagliflozin on the urinary albumin-to-creatinine ratio in patients with type 2 diabetes and established cardiovascular disease: an exploratory analysis from the EMPA-REG OUTCOME randomised, placebo-controlled trial". Lancet Diabetes and Endocrinology 5 (2017): 610621.

98. Wanner C., et al. "Empagliflozin and Progression of Kidney Disease in Type 2 Diabetes". The New England Journal of Medicine 375 (2016): 323-334.

99. Neuen BL., et al. "SGLT2 inhibitors for the prevention of kidney failure in patients with type 2 diabetes: a systematic review and meta-analysis". Lancet Diabetes and Endocrinology 7 (2019): 845-854.

100. Bilezikian JP., et al. "Evaluation of Bone Mineral Density and Bone Biomarkers in Patients With Type 2 Diabetes Treated
With Canagliflozin". The Journal of Clinical Endocrinology and Metabolism 101 (2016): 44-51.

101. Watts NB., et al. "Effects of Canagliflozin on Fracture Risk in Patients With Type 2 Diabetes Mellitus". The Journal of Clinical Endocrinology and Metabolism 101 (2016): 157-166.

102. Ljunggren Ö., et al. "Dapagliflozin has no effect on markers of bone formation and resorption or bone mineral density in patients with inadequately controlled type 2 diabetes mellitus on metformin". Diabetes, Obesity and Metabolism 14 (2012): 990-999.

103. Fioretto P., et al. "Long-Term Safety of Dapagliflozin in Older Patients with Type 2 Diabetes Mellitus: A Pooled Analysis of Phase IIb/III Studies". Drugs Aging 33 (2016): 511-522.

104. Kohler S., et al. "Safety and Tolerability of Empagliflozin in Patients with Type 2 Diabetes". Clinical Therapy 38 (2016): 1299-1313.

105. "SPC canagliflozin".

106. "SPC dapagliflozin".

107. "SPC empagliflozin".

108. "SPC ertugliflozin".

109. Azharuddin M., et al. "Sodium-glucose cotransporter 2 inhibitors and fracture risk in patients with type 2 diabetes mellitus: A systematic literature review and Bayesian network metaanalysis of randomized controlled trials". Diabetes Research and Clinical Practice 146 (2018): 180-190.

110. Ruanpeng D., et al. "Sodium-glucose cotransporter 2 (SGLT2) inhibitors and fracture risk in patients with type 2 diabetes mellitus: A meta-analysis". Diabetes Metabolism 33.6 (2017).

111. Tang HL., et al. "Lack of evidence for a harmful effect of sodium-glucose co-transporter 2 (SGLT2) inhibitors on fracture risk among type 2 diabetes patients: a network and cumulative meta-analysis of randomized controlled trials". Diabetes, Obesity and Metabolism 18 (2016): 1199-1206.

112. Qiu R., et al. "Canagliflozin: Efficacy and Safety in Combination with Metformin Alone or with Other Antihyperglycemic Agents in Type 2 Diabetes". Diabetes Therapy 7 (2016): 659678.

Citation: Robert Janknegt., et al. "SGLT-2 Inhibitors: Drug Selection by Means of the System of Objectified Judgement Analysis Method". Acta Scientific Pharmaceutical Sciences 5.7 (2021): 91-112. 
113. Fioretto P., et al. "Efficacy and safety of dapagliflozin, a sodium glucose cotransporter 2 (SGLT2) inhibitor, in diabetes mellitus". Cardiovascular Diabetology 14 (2015): 142.

114. Kohler S., et al. "Safety and Tolerability of Empagliflozin in Patients with Type 2 Diabetes". Clinical Therapy 38 (2016): 1299-1313.

115. Kohler S., et al. "Safety and Tolerability of Empagliflozin in Patients with Type 2 Diabetes: Pooled Analysis of Phase I-III Clinical Trials". Advances in Therapy 34 (2017): 1707-1726.

116. Davies MJ., et al. "Efficacy and safety of canagliflozin in patients with type 2 diabetes based on history of cardiovascular disease or cardiovascular risk factors: a post hoc analysis of pooled data". Cardiovascular Diabetology 16 (2017): 40.

117. Xiong W., et al. "Efficacy and safety of canagliflozin in patients with type 2 diabetes: A meta-analysis of randomized controlled trials". Medicine (Baltimore) 95 (2016): e5473.

118. Plosker GL. "Canagliflozin: a review of its use in patients with type 2 diabetes mellitus". Drugs 74 (2014): 807-824.

119. Li D., et al. "Risks of diabetic foot syndrome and amputation associated with sodium glucose co-transporter 2 inhibitors: A Meta-analysis of Randomized Controlled Trials". Diabetes Metabolism 44 (2018): 410-414.

120. Ryan PB., et al. "Comparative effectiveness of canagliflozin, SGLT2 inhibitors and non-SGLT2 inhibitors on the risk of hospitalization for heart failure and amputation in patients with type 2 diabetes mellitus: A real-world meta-analysis of 4 observational databases (OBSERVE-4D)". Diabetes, Obesity and Metabolism 20 (2018): 2585-2597.

121. Tang H., et al. "SGLT2 inhibitors and risk of cancer in type 2 diabetes: a systematic review and meta-analysis of randomised controlled trials". Diabetologia (2017).

122. Puckrin R., et al. "SGLT-2 inhibitors and the risk of infections: a systematic review and meta-analysis of randomized controlled trials". Acta Diabetology 55 (2018): 503-514.

123. Li D., et al. "Urinary tract and genital infections in patients with type 2 diabetes treated with sodium-glucose co-transporter 2 inhibitors: A meta-analysis of randomized controlled trials”. Diabetes, Obesity and Metabolism 19 (2017): 348-355.
124. Zaccardi F., et al. "Efficacy and safety of sodium-glucose cotransporter-2 inhibitors in type 2 diabetes mellitus: systematic review and network meta-analysis". Diabetes, Obesity and Metabolism 18 (2016): 783-794.

125. Bundhun PK and Huang F. "Adverse Drug Events Associated with sitagliptin Versus canagliflozin for the Treatment of Patients with Type 2 Diabetes Mellitus: A Systematic Comparison Through a Meta-Analysis". Diabetes Therapy 9 (2018): 18831895.

126. Meng Q., et al. "Efficacy of canagliflozin combined with antidiabetic drugs in treating type 2 diabetes mellitus: Meta-analysis of randomized control trials". Journal of Diabetes Investigation 7 (2016): 359-365.

127. Dawra VK., et al. "Effect of Rifampin on the Pharmacokinetics of Ertugliflozin in Healthy Subjects". Clinical Therapy 40 (2018): 1538-1547.

128. Zelniker TA., et al. "SGLT2 inhibitors for primary and secondary prevention of cardiovascular and renal outcomes in type 2 diabetes: a systematic review and meta-analysis of cardiovascular outcome trials". Lancet 393 (2019): 31-39.

Volume 5 Issue 7 July 2021

(C) All rights are reserved by Robert Janknegt., et al. 